\title{
Reflected planar Brownian motions, intertwining relations and crossing probabilities
}

\author{
Julien Dubédat \\ Université Paris-Sud, laboratoire de mathématiques bât. 425, 91405 Orsay Cedex, France \\ Received 25 March 2003; received in revised form 10 November 2003; accepted 16 January 2004 \\ Available online 12 May 2004
}

\begin{abstract}
Prompted by an example arising in critical percolation, we study some reflected Brownian motions in symmetric planar domains and show that they are intertwined with one-dimensional diffusions. In the case of a wedge, the reflected Brownian motion is intertwined with the 3-dimensional Bessel process. This implies some simple hitting distributions and sheds some light on the formula proposed by Watts for double-crossing probabilities in critical percolation.
\end{abstract}

(c) 2004 Elsevier SAS. All rights reserved.

\section{Résumé}

Généralisant un exemple apparaissant en percolation critique, nous étudions certains mouvements browniens réfléchis dans des domaines plans symétriques et montrons qu'ils sont entrelacés avec des diffusions réelles. Dans le cas d'un cône, ce mouvement brownien réfléchi est entrelacé avec le processus de Bessel de dimension 3. Ceci implique certaines distributions de point de sortie simples et des résultats en relation avec la formule proposée par Watts pour les probabilités de croisement double en percolation critique.

(c) 2004 Elsevier SAS. All rights reserved.

MSC: 60J99; 82B43

Keywords: Reflected Brownian motion; Critical percolation; Intertwining relations; Watts' formula

\section{Introduction and notations}

It has been pointed out in $[10,20]$ that the hitting distribution of a certain reflected Brownian motion (RBM) in an equilateral triangle was uniform. More precisely, if the Brownian motion is started from one corner and reflected on the two adjacent sides with the oblique reflection angle $\pi / 6$ away from the normal direction toward the opposite side, then the Brownian motion will hit this opposite side with uniform distribution. The proof uses a discrete version of this result (reflected simple random walk) on a well-chosen triangular lattice, and an invariance principle (i.e. the reflected random walk converges to reflected Brownian motion). This result is then combined

E-mail address: julien.dubedat @math.u-psud.fr (J. Dubédat). 
with a locality property in order to identify the law of the whole "hull" of this stopped reflected Brownian motion with that of a chordal $\mathrm{SLE}_{6}$ process (that has the same uniform hitting distribution and has also a locality property).

In [16], Smirnov proved that $\mathrm{SLE}_{6}$ is the scaling limit of critical percolation cluster interfaces on the triangular lattice. The main step in this proof is the derivation of the fact that in the scaling limit, the above-mentioned hitting distribution (if one replaces the RBM by the percolation interface) becomes uniform. It makes an important use of some specific features of the triangular lattice. Note that in the case of critical percolation, the discrete hitting probabilities are not uniform, as opposed to those for the reflected simple random walk.

One of the motivations of the present paper is to see whether one can generalize this hitting probability property of reflected Brownian motion to other symmetric shapes (for instance other wedges) and other reflection angles. It turns out that one can choose the reflection angles in such a way that hitting probabilities of segments orthogonal to the symmetry axis of the shape remain uniform. Furthermore, this fact is closely related to a relation between these reflected Brownian motions and one-dimensional diffusions. More precisely, the projection of the RBM on the symmetry axis is a diffusion in its own filtration; for wedges, one recovers the 3-dimensional Bessel process. This provides a new example of non-trivial intertwining relations (see [14,7]; for other examples of intertwinings, see [5]). Our proofs rely also on reflected simple random walks and an invariance principle. Other studies of reflected Brownian motion in cones include $[17,11,4]$.

We shall see that the formula proposed by Watts [19] for double-crossing probabilities (i.e. simultaneous topto-bottom and left-to-right crossing of a quadrilateral) for critical percolation and for which it is not clear that the $\mathrm{SLE}_{6}$ approach will confirm it, is in fact satisfied (in some appropriate sense) by the reflected Brownian motion; this will follow from the study of time-reversed reflected Brownian motions.

This paper is organized as follows. First, we define some reflected random walks in wedges and study their scaling limit. Then we prove that the limiting reflected Brownian motions are intertwined with the 3-dimensional Bessel process. Making use of this fact, we determine the time reversals of these RBMs, and discuss an analogue of Watts' formula. Finally, we generalize some of the results to symmetric shapes ("vases"), essentially replacing the 3-dimensional Bessel process by a more general one-dimensional diffusion.

\section{Invariance principle}

Let $\alpha \in\left(0, \frac{\pi}{2}\right)$. We will consider the wedge

$$
C_{\alpha}=\{z \in \mathbb{C}:-\alpha \leqslant \arg z \leqslant \alpha\} \subset \mathbb{C}
$$

and the rectangular lattice

$$
L_{\alpha}=(\cos \alpha) \mathbb{Z}+i(\sin \alpha) \mathbb{Z} .
$$

We will study random walks on the graph $\Gamma_{\alpha}=L_{\alpha} \cap C_{\alpha}$.

In this section, the angle $\alpha$ is fixed, so we will drop the subscript $\alpha$. Consider the following random walk on the graph $\Gamma=L \cap C$ : for an inner point $x$, the transition probability is:

$$
\left\{\begin{array}{l}
p=p(x, x+\cos \alpha)=p(x, x-\cos \alpha)=\frac{\sin ^{2} \alpha}{2}, \\
q=p(x, x+i \sin \alpha)=p(x, x-i \sin \alpha)=\frac{\cos ^{2} \alpha}{2},
\end{array}\right.
$$

so that such a step has a zero mean, and its covariance matrix is a multiple of the identity. If $x \neq 0$ is a boundary point with $\arg (x)=\alpha$, the transition probability is:

$$
\left\{\begin{array}{l}
p(x, x+\cos \alpha)=p(x, x+\cos \alpha+i \sin \alpha)=\frac{\sin ^{2} \alpha}{2}, \\
p(x, x-i \sin \alpha)=p(x, x)=\frac{\cos ^{2} \alpha}{2} .
\end{array}\right.
$$

Notice that in this case,

$$
\arg \left(\mathbb{E}^{x}\left(X_{1}-x\right)\right)=2 \alpha-\frac{\pi}{2} .
$$




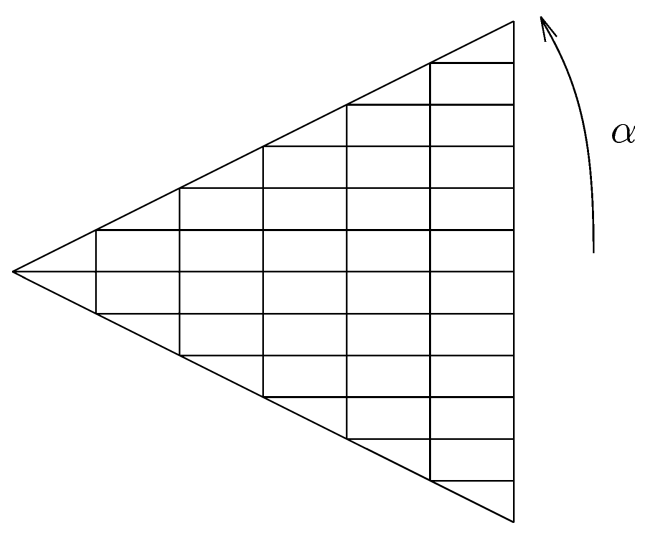

Fig. 1. A wedge with its associated rectangular lattice.
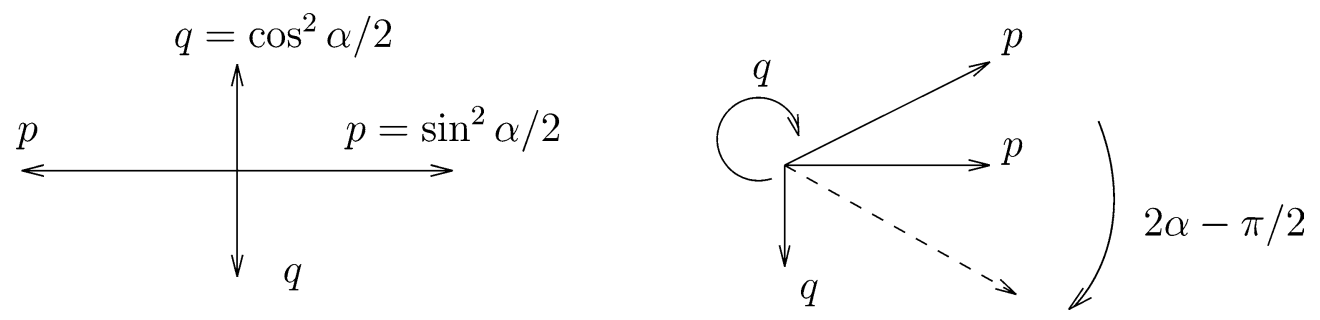

Fig. 2. Transition probabilities for an inner point and a boundary point.

On the other boundary line, the transition probabilities are defined symmetrically.

Finally for the apex, set

$$
r=p(0, \cos \alpha)=p(0, \cos \alpha \pm i \sin \alpha)=\frac{1-p(0,0)}{3}>0 .
$$

The exact value of this positive probability will not matter in the continuous limit. This random walk starting from $x \in \Gamma$ will be denoted by $\left(X_{n}^{x}\right)_{n} \geqslant 0$. We will also call $\left(Y_{t}^{x}\right)_{t \geqslant 0}$ the Brownian motion in $C$ starting from $x$ and reflected on the boundary with angle $-\alpha$ (see [17] for a definition of this process; we use the same conventions for reflection angles, i.e. positive angles point towards the apex).

The following result is classical. We include a proof for the sake of completeness (and in order to point out later how it can be generalized).

Lemma 1. Let $x$ be a point in $C$, and $\left(x_{n}\right)$ a sequence in $\Gamma$ such that $\left|x_{n}-n x\right| \leqslant 1 / 2$ for all $n \in \mathbb{N}$. Then the following weak convergence of processes holds as $n$ goes to infinity:

$$
\left(\frac{1}{n} X_{\left\lfloor n^{2} t / \sin ^{2} \alpha \cos ^{2} \alpha\right\rfloor}^{x_{n}}\right)_{t \geqslant 0} \rightarrow\left(Y_{t}^{x}\right)_{t \geqslant 0}
$$

Proof. Let $\left(Y_{t}^{(n)}\right)$ be a continuous $C$-valued process that interpolates linearly the process:

$$
\left(\frac{1}{n} X_{\left\lfloor n^{2} t / \sin ^{2} \alpha \cos ^{2} \alpha\right\rfloor}^{x_{n}}\right)
$$


Choose a Hölder exponent $\beta, 0<\beta<\frac{1}{2}$. Then it is standard to check that for any fixed $T>0$, the following estimate on $\beta$-Hölder norms holds:

$$
\lim _{K \rightarrow \infty} \limsup _{n \in \mathbb{N}} \mathbb{P}\left(\left\|Y^{(n)}\right\|_{[0, T], \beta} \geqslant K\right)=0 .
$$

One proceeds exactly as in the proof of the a.s. $\beta$-Hölder continuity for Brownian paths (there is no particular problem due to the boundary here). Hence we get a tight sequence in the Polish space of $\beta$-Hölder continuous mappings from $[0, T]$ to $C$. Using Prohorov's theorem, one gets the existence of subsequential weak limits. Then a standard diagonal argument yields subsequential weak limits for the whole process in the Wiener space of $C$-valued processes.

So we have to check that there is only one possible weak limit, namely the reflected Brownian motion $\left(Y_{t}\right)$. To prove this, one can use the submartingale problem characterization of reflected BM ([17], Theorem 2.1). More precisely, the RBM is the only $C$-valued process starting from $x$ such that for any $f \in C_{b}^{2}(C)$ (twice differentiable with bounded derivatives) with positive derivatives on the boundary along the reflection direction, the real valued process:

$$
f\left(Y_{t}\right)-\frac{1}{2} \int_{0}^{t} \Delta f\left(Y_{s}\right) d s
$$

is a submartingale. So let $f$ be such a function; it is sufficient to check that for any $0 \leqslant s<t$ :

$$
\liminf _{n \in N} \mathbb{E}_{(n)}\left(f\left(Y_{t}\right)-f\left(Y_{s}\right)-\frac{1}{2} \int_{s}^{t} \Delta f\left(Y_{u}\right) d u\right) \geqslant 0
$$

where $\mathbb{E}_{(n)}$ designates the expectation operator for the $n$th approximate process. Consider the stopping time $\tau=\inf \left(t \geqslant 0:\left|Y_{t}^{x}\right| \geqslant M\right)$ for some large number $M$. It is enough to check the submartingale inequalities up to time $\tau$. Notice that the following Taylor expansion holds:

$$
\begin{aligned}
& (p f(x+\cos \alpha / n)+p f(x-\cos \alpha / n)+q f(x+i \sin \alpha / n)+q f(x-i \sin \alpha / n)) \\
& \quad=\frac{1}{2 n^{2}} \sin ^{2} \alpha \cos ^{2} \alpha \Delta f(x)+o\left(\frac{1}{n^{2}}\right)
\end{aligned}
$$

and that the error term is uniform in $x \in C \cap D(0, M)$ (using for instance Hadamard's lemma). Let $u_{k}=$ $k \sin ^{2} \alpha \cos ^{2} \alpha / n^{2}, k \in \mathbb{N}$, correspond to the discrete jump times, and let $B$ be the boundary of $C$. Then:

$$
\begin{aligned}
\mathbb{E}_{(n)}^{x}\left(f\left(Y_{t \wedge \tau}\right)-f\left(Y_{S \wedge \tau}\right)\right) & =\mathbb{E}_{(n)}^{x}\left(\sum_{s \wedge \tau \leqslant u_{k}<t \wedge \tau} f\left(Y_{u_{k+1}}\right)-f\left(Y_{u_{k}}\right)\right) \\
& =\mathbb{E}_{(n)}^{x}\left(\sum_{s \wedge \tau \leqslant u_{k}<t \wedge \tau} \frac{1}{2 n^{2}} \cos ^{2} \alpha \sin ^{2} \alpha \Delta f\left(Y_{u_{k}}\right)+\varepsilon\left(Y_{u_{k}}\right) \mathbb{1}_{Y_{u_{k}} \in B}\right)+\mathrm{o}(1) \\
& =\mathbb{E}_{(n)}^{x}\left(\int_{s \wedge \tau}^{t \wedge \tau} \frac{1}{2} \Delta f\left(Y_{u}\right) d u\right)+\mathrm{o}(1)+\sum_{y \in B} \mathbb{E}_{(n)}^{x}\left(L_{y}\right) \varepsilon(y) .
\end{aligned}
$$

The error term o(1) is simply a Riemann sum error. Then there is a "boundary error term". In the formula, $L_{y}$ designates the local time at $y$ (number of discrete jumps to $y$ ) between times $s \wedge \tau$ and $t \wedge \tau$, and $\varepsilon(y)$ is defined as (if $\arg (y)=\alpha$ for instance):

$$
\varepsilon(y)=q(f(y)-f(y+i \sin \alpha / n))+p\left(f\left(y+e^{i \alpha} / n\right)-f(y-\cos \alpha / n)\right) .
$$




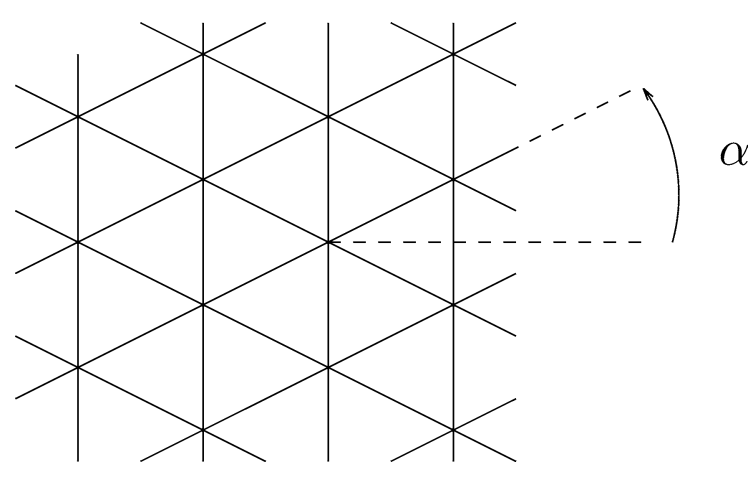

Fig. 3. "Stretched" triangular lattice.

The first order term is proportional to the derivative of $f$ at $y$ along the reflection direction, hence is positive by hypothesis. One may write: $\varepsilon(y)+\mathrm{O}\left(1 / n^{2}\right) \geqslant 0$ for $y \in B$, the error term $\mathrm{O}\left(1 / n^{2}\right)$ being uniform in $y \in C \cap D(0, M)$. So the only thing to check now is that the time spent on the boundary is negligible, more precisely:

$$
\sum_{y \in B} \mathbb{E}_{(n)}^{x}\left(L_{y}\right)=\mathrm{o}\left(n^{2}\right)
$$

Recall that we consider the local time between times $s$ and $t$, and before exit time $\tau$; one may drop this last condition for the sake of simplicity. Justifying the very intuitive fact that the walk spends a negligible time on a negligible part of the state space is rather tedious. The discrete intertwining relations allow explicit computations that yield the result when the starting point is the apex 0 (see below). Then, using the Markov property, one sees it is enough to prove this estimate starting from the apex. This concludes the proof.

One may carry out the same proof with different transition probabilities on the boundary, corresponding to different reflection angles; to derive the final local time estimate in the general case, one may use the local Central Limit Theorem.

Remark 1. Similar results hold for different lattices, in particular "stretched triangular lattices", i.e. images of the standard triangular lattice under an orthogonal affinity. In the special case $\alpha=\pi / 6$, one may use the standard triangular lattice, with the usual transition probability for inner point (i.e., $1 / 6$ for each neighbour). In the case $\alpha=\pi / 4$, these two approaches give the same lattice, namely the square lattice.

Note that all these reflected Brownian motions have the same parameter $\alpha=-1$ in Varadhan-Williams conventions [17] (what we call $\alpha$ would be denoted $\xi / 2$ in [17]).

\section{Intertwining relations}

First, let us recall the notion of intertwined Markovian semigroups (for some background on the subject, see [7]). Let $\left(P_{t}, t \geqslant 0\right)$ and $\left(Q_{t}, t \geqslant 0\right)$ be two Markovian semigroups with respective state spaces $\left(S_{P}, \mathcal{S}_{P}\right)$ and $\left(S_{Q}, \mathcal{S}_{Q}\right)$, and $\Lambda$ a Markov transition kernel from $\left(S_{Q}, \mathcal{S}_{Q}\right)$ to $\left(S_{P}, \mathcal{S}_{P}\right)$. The two semigroups are said to be intertwined by $\Lambda$ if the following identity of Markov transition kernels from $S_{Q}$ to $S_{P}$ holds:

$$
\Lambda P_{t}=Q_{t} \Lambda
$$

for all $t \geqslant 0(t$ is either a discrete or a continuous time parameter). 
Suppose that there exists a measurable function $\phi: S_{P} \rightarrow S_{Q}$ such that $\Lambda\left(z, \phi^{-1}(z)\right)=1$ for all $z \in S_{Q}$. Then, as shown in [14], if $\left(Y_{t}\right)$ is a Markov process with semigroup $\left(P_{t}\right)$ and initial law $\Lambda\left(z_{0},.\right)$ for some $z_{0} \in S_{Q}$, and $Z_{t}=\phi\left(Y_{t}\right)$, then $\left(Z_{t}\right)$ is a Markov process with semigroup $\left(Q_{t}\right)$ and starting state $z_{0}$. Moreover, for any fixed time $t$, the following filtering formula holds:

$$
\mathbb{P}\left(Y_{t} \in . \mid\left(Z_{s}\right)_{0 \leqslant s \leqslant t}\right)=\Lambda\left(Z_{t}, .\right) .
$$

Under appropriate regularity conditions (which will be satisfied in all examples that we shall consider here), this formula also holds for almost surely finite stopping times $T$ (w.r. to the filtration of $Z$ ). In particular, for $T=T_{z}$, the first hitting time of $z$ by $Z$, if $T_{z}<\infty$ a.s., then the random variable $Y_{T_{z}}$ has distribution $\Lambda(z,$.$) and is independent$ of $\left(Z_{s}\right)_{0 \leqslant s \leqslant T_{z}}$.

Consider as before the random walk $\left(X_{n}\right)$ on the graph $\Gamma$. Let $\Lambda$ be the following Markov transition kernel from $\mathbb{N}$ to $\Gamma$ (we mention that these discrete spaces are equipped with discrete $\sigma$-algebras):

$$
\Lambda(x, .)=\frac{1}{2 x+1} \sum_{k=-x}^{x} \delta_{x} \cos \alpha+k i \sin \alpha .
$$

Let $\left(P_{n}\right)$ be the semigroup of $\left(X_{n}\right)$, and let $\left(Q_{n}\right)$ be the semigroup of the random walk on $\mathbb{N}$ with transition probability:

$$
\left\{\begin{array}{l}
q(i, i-1)=\frac{\sin ^{2} \alpha}{2} \frac{2 i-1}{2 i+1} \\
q(i, i)=\cos ^{2} \alpha \\
q(i, i+1)=\frac{\sin ^{2} \alpha}{2} \frac{2 i+3}{2 i+1}
\end{array}\right.
$$

for $i>0$ and $q(0,0)=1-3 r, q(0,1)=3 r$. Note that $i \mapsto \frac{1}{2 i+1}$ is harmonic for this transition kernel, except at 0 . Then, inspection of the transition probabilities shows that the following intertwining relation holds:

$$
\Lambda P_{n}=Q_{n} \Lambda
$$

for all $n \geqslant 0$.

Let $\left(Y_{t}\right)$ be the reflected Brownian motion in the wedge $C$ with reflection angle $-\alpha$. Let $\left(P_{t}\right)_{t \geqslant 0}$ be its semigroup. Let $\left(Q_{t}\right)_{t \geqslant 0}$ be the generator of the 3-dimensional Bessel process (with values in $\mathbb{R}_{+}$), and $\Lambda$ be the Markov transition kernel from $\mathbb{R}_{+}$to $C$ such that $\Lambda(x,$.$) is the uniform distribution on the segment$ $[x-i \tan \alpha, x+i \tan \alpha]$.

In the scaling limit, the previous intertwining leads to:

Proposition 1. The semigroups $P$ and $Q$ are intertwined by the Markov kernel $\Lambda$. In particular, for $z \geqslant 0$, $Z_{t}=\Re Y_{t}^{\Lambda(z, .)}$ is a 3-dimensional Bessel process starting from $x$.

Proof. It is obvious that the intertwining relation holds in the limit. The only thing to check is that the intertwined random walk on $\mathbb{N}$ converges to a 3-dimensional Bessel process. One may proceed as in the previous proof, after computing:

$$
\begin{aligned}
& \left(\cos ^{2} \alpha f(x)+\frac{\sin ^{2} \alpha}{2}\left(\frac{2 n x / \cos \alpha+3}{2 n x / \cos \alpha+1} f(x+\cos \alpha / n)+\frac{2 n x / \cos \alpha-1}{2 n x / \cos \alpha+1} f(x-\cos \alpha / n)\right)\right)-f(x) \\
& =\frac{\sin ^{2} \alpha \cos ^{2} \alpha}{n^{2}}\left(\frac{1}{x} f^{\prime}(x)+\frac{1}{2} f^{\prime \prime}(x)\right)+o\left(1 / n^{2}\right) .
\end{aligned}
$$

Here one recognizes the generator of the 3-dimensional Bessel process (the factor $\frac{\sin ^{2} \alpha \cos ^{2} \alpha}{n^{2}}$ corresponds to the time scaling). Such discrete approximations of the 3-dimensional Bessel process are classical (see [12]). 
The Markov transition kernel $\Lambda$ acts on bounded Borel functions in the following way: if $f$ is a bounded Borel function on $C$, then $\Lambda f$ is a bounded Borel function on $\mathbb{R}_{+}$such that:

$$
(\Lambda f)(x)=\mathbb{E}(f(x+i U \tan \alpha))
$$

where $U$ is a uniform random variable on $[-1,1]$. One may remark that these intertwining relations fit in the "filtering type framework" described in [7].

As Jim Pitman pointed out to us, there is an analogy with the situation in his $(2 M-X)$ theorem: let $B$ be a standard (real) Brownian motion starting from 0 , and let $M_{t}$ be its supremum up to time $t$. Then the process $\left(2 M_{t}-X_{t}\right)_{t \geqslant 0}$ is a 3-dimensional Bessel process (in its own filtration) (see [14,13]). A proof of this fact involves an intertwining relation. More precisely, note $Y_{t}=M_{t}-B_{t}, Z_{t}=2 M_{t}-B_{t}$. Then, if $\left(\mathcal{G}_{t}\right)_{t \geqslant 0}$ designates the natural filtration of $\left(Z_{t}\right)_{t \geqslant 0}$, the following relation holds for every Borel function $f: \mathbb{R}_{+} \rightarrow \mathbb{R}_{+}$:

$$
\mathbb{E}\left(f\left(Y_{t}\right) \mid \mathcal{G}_{t}\right)=\int_{0}^{1} f\left(x Z_{t}\right) d x .
$$

The Markov kernel in this last situation: $\Lambda f(z)=\int_{0}^{1} f(x z) d x$ is very similar to the one we described earlier.

The Brownian motion in a plane strip $\{z: 0 \leqslant \Im z \leqslant 1\}$ with normal reflection on the boundary may be seen as a degenerate limiting case when $\alpha \searrow 0$. Indeed, the reflection direction makes an angle $\pi / 2-\alpha$ with the boundary, and the two boundary half-lines make an angle $2 \alpha$. This particular RBM may be represented as $Z=X+i f(Y)$, where $X$ and $Y$ are independent standard (one-dimensional) BMs, and $f$ is the "seesaw" function: for all $k \in \mathbb{Z}$,

$$
\begin{aligned}
& f(x)=x-2 k \quad \text { if } 2 k \leqslant x \leqslant 2 k+1, \\
& f(x)=2 k+2-x \quad \text { if } 2 k+1 \leqslant x \leqslant 2 k+2 .
\end{aligned}
$$

In this case, the intertwining relation is easily proved. Indeed, let $\phi$ be any bounded Borel function on the strip and $t$ be a fixed positive time. We have to check that:

$$
\mathbb{E}_{x}\left(\phi\left(X_{t}+i U\right)\right)=\mathbb{E}\left(\mathbb{E}_{x+i U}\left(\phi\left(Z_{t}\right)\right)\right)
$$

where $U$ is an independent uniform random variable. Since the real and the imaginary part of $Z$ are independent, we have only to check that, for any bounded Borel function $\varphi$ on $[0,1]$ :

$$
\mathbb{E}(\varphi(U))=\mathbb{E}\left(\mathbb{E}_{U}\left(\varphi\left(f\left(Y_{t}\right)\right)\right)\right)=\mathbb{E}_{0}\left(\varphi\left(f\left(U+Y_{t}\right)\right)\right)
$$

which is readily seen (if $V$ is any symmetric r.v. independent from $U, f(U+V)$ is a uniform r.v.).

Remark 2. In the general case, the intertwining relation does not seem obvious directly in the continuous setting, hence the use of discrete approximations. Also, the real part of the RBM started from a fixed, inner point (not the apex) does not appear to have similar properties; in this case, the relation between the filtration of the RBM and the filtration of its real part seems very intricate, and the real part is generically not Markovian in its own filtration.

\section{Time reversal}

In this section, we consider the time reversals of the reflected Brownian motions studied in the previous sections. The intertwining relation enables an explicit computation of discrete Green functions, hence the determination of the time reversal of discrete random walks; then one takes the continuous limit.

Proposition 2. The time reversal of the RBM with reflection angle $-\alpha$ starting from 0 and stopped when hitting $\{z: \Re z \geqslant x\}$ is the RBM with reflection angle $+\alpha$ starting from the uniform distribution on the segment 
$[x-i \tan \alpha ; x+i \tan \alpha]$, conditioned not to hit $\{z: \Re z \geqslant x\}$ again, and killed at 0. This last process is intertwined with a 3-dimensional Bessel process, with intertwining kernel $\Lambda:(\Lambda f)(x)=\int_{-1}^{1} f(x+i u \tan \alpha) d u / 2$.

Loosely speaking, the time reversal of a reflected Brownian motion in $C_{\alpha}$ with reflection angle $-\alpha$ starting from 0 is the reflected Brownian motion reflected at angle $+\alpha$, killed at 0 , starting from "the uniform distribution at infinity".

Proof. Recall that we considered a random walk $X$ on $\Gamma$ intertwined with a random walk $Y$ on $\mathbb{N}$. Let $G$ be the Green function for $X$ killed when it hits $\{z: \Re z \geqslant N \cos \alpha\}$ for some large $N$. Let $\tilde{G}$ be the Green function for $Y$ killed at level $N$. Then it is obvious from the intertwining of $X$ and $Y$ that:

$$
G(0, x \cos \alpha+i y \sin \alpha)=\frac{1}{2 x+1} \tilde{G}(0, x)
$$

where $0 \leqslant x \leqslant N,-x \leqslant y \leqslant x, x, y \in \mathbb{N}$. As previously mentioned, the function $i \mapsto \frac{1}{2 i+1}$ is harmonic for the Markov kernel of $Y$ (except in 0 ). From this, it is easy to compute:

$$
\tilde{G}(0, y)=\frac{(2 y+1)\left(1-\frac{2 y+1}{2 N+1}\right)}{\cos ^{2} \alpha} .
$$

Then Nagasawa's formula (see e.g. [15], III.42) yields the Markov kernels of the time reversals $\hat{X}$ and $\hat{Y}$ of $X$ and $Y$. For instance, we record the transition probability for $\hat{X}$, if $x \in \Gamma$ is an inner point:

$$
\left\{\begin{array}{l}
\hat{p}(x, x+i \sin \alpha)=\hat{p}(x, x-i \sin \alpha)=\frac{\cos ^{2} \alpha}{2}, \\
\hat{p}(x, x-\cos \alpha)=\frac{\sin ^{2} \alpha}{2} \frac{N-x+1}{N-x} \\
\hat{p}(x, x+\cos \alpha)=\frac{\sin ^{2} \alpha}{2} \frac{N-x-1}{N-x}
\end{array}\right.
$$

Notice that, as $N$ goes to infinity, one gets the original transition probabilities. On the boundary, the reflection angle is reversed, which is not surprising. At this point, one takes the continuous limit as in Section 1 (obviously the time reversal operation is compatible with the continuous limit). The various claims follow easily.

The conformal equivalence $z \mapsto z^{\beta}$ maps $C_{\alpha}$ onto $C_{\beta \alpha}$. After an appropriate time change, this yields a general result for the time reversal of a reflected Brownian motion in an infinite wedge.

In the set-up of the proposition, one may notice that up to its first hitting of the boundary, the time-reversed RBM has the law of a Brownian excursion in the half-plane $\{z: \Re z \leqslant x\}$ (for background on Brownian excursions, see $[10,18])$. One may use this to compute some probabilities of (indirect) interest in critical percolation. For simplicity, we will consider a RBM $Z$ in the cone $\Delta=(1 / 2+i \sqrt{3} / 2)-i C_{\pi / 6}$ starting from the apex and stopped on hitting the real line at time $\tau$ (we are looking at a RBM in an equilateral triangle). We have seen that $Z_{\tau}$ is uniformly distributed on $[0,1]$. Let $g$ be the last time spent by $Z$ on the boundary before $\tau$. We are interested in the joint law $\left(Z_{g}, Z_{\tau}\right)$, or rather in which side was last visited by $Z$ conditionally on the exit point $Z_{\tau}$; so we will consider the event:

$$
\mathcal{R}=\left\{\Re Z_{g} \geqslant 1 / 2\right\} .
$$

Since the time reversal of $Z$ is a Brownian excursion until it hits the boundary, we have to compute the "harmonic measure" for the Brownian excursion.

Let $Y$ be a Brownian excursion in $\mathbb{H}=\{z: \Im z \geqslant 0\}$, and let $T$ be the first time it hits $\partial \Delta$. Let $\varepsilon>0, M>0$, and $B$ a complex Brownian motion exiting the strip $\{z: 0<\Im z<M\}$ at time $T_{M}$. As $M$ goes to infinity, the Brownian motion $B$ conditioned on exiting the strip by the top converges to the Brownian excursion. Hence, making use of the Markov property of $B$ : 


$$
\begin{aligned}
\mathbb{P}_{x+i \varepsilon}\left(Y_{T} \in d y\right) & =\lim _{M \rightarrow \infty} \mathbb{P}_{x+i \varepsilon}\left(B_{T} \in d y \mid \Im B_{T_{M}}=M\right) \\
& =\mathbb{P}_{x+i \varepsilon}\left(B_{T} \in d y\right) \lim _{M \rightarrow \infty} \frac{\mathbb{P}_{y}\left(\Im B_{T_{M}}=M\right)}{\mathbb{P}_{x+i \varepsilon}\left(\Im B_{T_{M}}=M\right)} \\
& =\frac{\Im}{\varepsilon} \mathbb{P}_{x+i \varepsilon}\left(B_{T} \in d y\right) .
\end{aligned}
$$

It is well known that harmonic measure is conformally invariant (see e.g. [13]), and that the harmonic measure on the real line seen from $a+i b$ is given by a Cauchy law (see e.g. [15]) with density:

$$
\frac{b d x}{\pi\left(b^{2}+(x-a)^{2}\right)} .
$$

Moreover, according to the Schwarz-Christoffel formula (see [2]), the holomorphic map:

$$
F(z)=\int_{0}^{z} u^{-2 / 3}(1-u)^{-2 / 3} d u / B(1 / 3,1 / 3)
$$

is the conformal equivalence between the upper half-plane $\mathbb{H}$ and the equilateral triangle $\Delta \cap \mathbb{H}$ that maps $(0,1, \infty)$ to $(0,1,1 / 2+i \sqrt{3} / 2)$. If $a \in(0,1)$ is such that $F(a)=x$, we see that:

$$
P_{x}\left(Y_{T} \in d y\right)=\Im y \frac{d F^{-1}(y)}{\pi F^{\prime}(a)\left(F^{-1}(y)-a\right)^{2}} .
$$

Then, one may compute:

$$
\begin{aligned}
\mathbb{P}\left(\mathcal{R} \mid Z_{\tau} \in d x\right) & =F^{\prime}(a)^{-1} \int_{1}^{\infty} \frac{d t}{\pi(t-a)^{2}} \int_{1}^{t} \frac{(u(u-1))^{-2 / 3}}{B(1 / 3,1 / 3)} \frac{\sqrt{3}}{2} d u \\
& =F^{\prime}(a)^{-1} \frac{\sqrt{3}}{2 \pi B(1 / 3,1 / 3)} \int_{1}^{\infty} \frac{d u}{(u(u-1))^{2 / 3}(u-a)} \\
& =\frac{\pi \sqrt{3}}{3 \Gamma(2 / 3)^{3}}(a(1-a))^{2 / 3}{ }_{2} F_{1}(1,4 / 3 ; 5 / 3 ; a) \\
& =\frac{1}{B(2 / 3,2 / 3)} \int_{0}^{a} \frac{d t}{(t(1-t))^{1 / 3}},
\end{aligned}
$$

where ${ }_{2} F_{1}(1,4 / 3 ; 5 / 3 ;$.) designates a hypergeometric function (see e.g. [3] or [1], especially formulas 15.3 .1 and 15.2.5).

\section{Relation with Watts' formula}

In this section, we recall Watts' formula [19] and explain how it may be translated in the SLE language. This relation was suggested to us by Wendelin Werner. We shall see that it is closely related with the formula we derived above; in fact, this is one of the initial motivations for the present paper.

Recall that Cardy's formula [6] gives the asymptotic behaviour of the probability of an open crossing between two sides of a topological rectangle in the limit when the mesh of the lattice goes to zero (for critical percolation). This is equivalent to the fact that the hitting distribution of the exploration process in an equilateral triangle is 

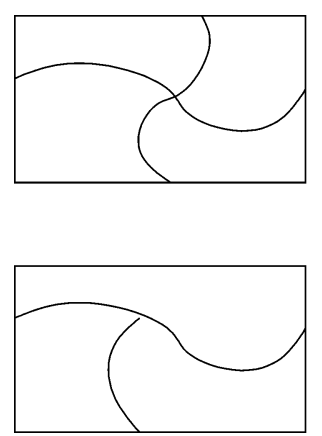
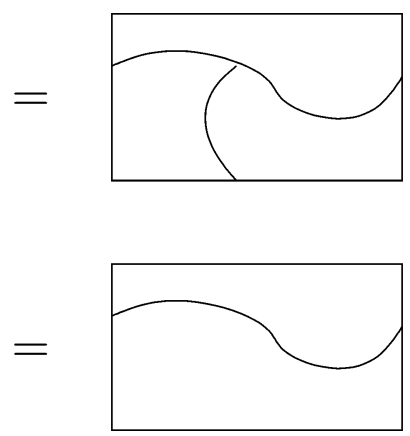

Fig. 4. Paths in a topological rectangle.
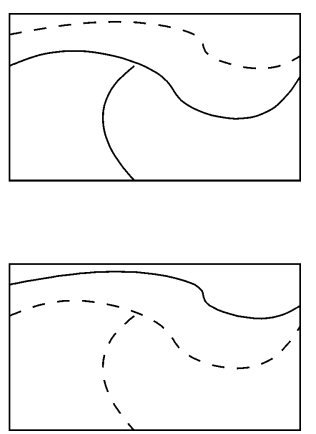

uniform. This was shown by Smirnov [16] to hold in the case of site percolation on the triangular lattice, and it follows that the scaling limit of the whole exploration process is $\mathrm{SLE}_{6}$. Note that it is not difficult (see [9]) to prove directly in the continuous setting that this hitting distribution for $\mathrm{SLE}_{6}$ is uniform.

Trying to generalize Cardy's results using the same approach based on conformal field theory, Watts [19] considered the event that there exists simultaneously an open left-to-right crossing and an open top-to-bottom crossing of a topological rectangle. He proposed a formula to describe the asymptotic behaviour of the probability of this event when the mesh of the lattice vanishes, that seems to fit well with numerical simulations. Just as in the case of Cardy's formula, the double-crossing event can in fact be rephrased in terms of the exploration process.

For simplicity, we will discuss critical percolation on the triangular lattice (so each vertex is colored in black with probability $p=1 / 2$ ). Let $R$ be a topological rectangle, its boundary consisting of four disjoint arcs: $\partial_{L}$, $\partial_{T}, \partial_{R}, \partial_{B}$ (left, top, right, bottom). Let $C\left(\partial_{i}, \partial_{j}, c\right)$ be the event that there exists a crossing between $\partial_{i}$ and $\partial_{j}$ with color $c$ (here $i, j \in\{L, T, R, B\}$, and the color $c$ is black or white: $c \in\{b, w\}$ ). We will also need the event $T\left(\partial_{i}, c\right)$ that there exists a connected component with color $c$ linking the four boundary arcs except maybe $\partial_{i}$. As the triangular lattice is self-matching (see [8]), it is classical that the two events $C\left(\partial_{L}, \partial_{R}, w\right)$ and $C\left(\partial_{T}, \partial_{B}, b\right)$ are complementary:

$$
C\left(\partial_{L}, \partial_{R}, w\right)=\overline{C\left(\partial_{T}, \partial_{B}, b\right)} .
$$

Now we are interested in $C\left(\partial_{L}, \partial_{R}, b\right) \cap C\left(\partial_{T}, \partial_{B}, b\right)$. With a little plane topology, one sees that:

$$
\begin{aligned}
& C\left(\partial_{L}, \partial_{R}, b\right) \cap C\left(\partial_{T}, \partial_{B}, b\right)=T\left(\partial_{T}, b\right) \backslash C\left(\partial_{L}, \partial_{R}, w\right), \\
& T\left(\partial_{T}, b\right)=C\left(\partial_{L}, \partial_{R}, b\right) \backslash T\left(\partial_{T}, w\right) .
\end{aligned}
$$

Fig. 4 illustrates these relations.

Translating this into probabilities:

$$
\mathbb{P}\left(C\left(\partial_{L}, \partial_{R}, b\right) \cap C\left(\partial_{T}, \partial_{B}, b\right)\right)=\mathbb{P}\left(T\left(\partial_{T}, b\right)\right)-\mathbb{P}\left(T\left(\partial_{T}, b\right) \cap C\left(\partial_{L}, \partial_{R}, w\right)\right)
$$

and

$$
\mathbb{P}\left(T\left(\partial_{T}, b\right)\right)=\mathbb{P}\left(C\left(\partial_{L}, \partial_{R}, b\right)\right)-\mathbb{P}\left(C\left(\partial_{L}, \partial_{R}, b\right) \cap T\left(\partial_{T}, w\right)\right) .
$$

Keeping in mind that each vertex is colored with probability $1 / 2$, so that changing all the colors is measurepreserving, one gets:

$$
\mathbb{P}\left(C\left(\partial_{L}, \partial_{R}, b\right) \cap C\left(\partial_{T}, \partial_{B}, b\right)\right)=\mathbb{P}\left(C\left(\partial_{L}, \partial_{R}, b\right)\right)-2 \mathbb{P}\left(C\left(\partial_{L}, \partial_{R}, w\right) \cap T\left(\partial_{T}, b\right)\right) .
$$

Consider now the situation in an equilateral triangle $A B C$. Let $X$ be the rightmost point on $B C$ that is separated from $A C$ by a black path. Cardy's formula tells that $X$ is uniformly distributed on $B C$ (see Fig. 5). The topological rectangle $A D X E$ delimited by the upper-half of $A B$, the rightmost black path (solid), the leftmost white path 


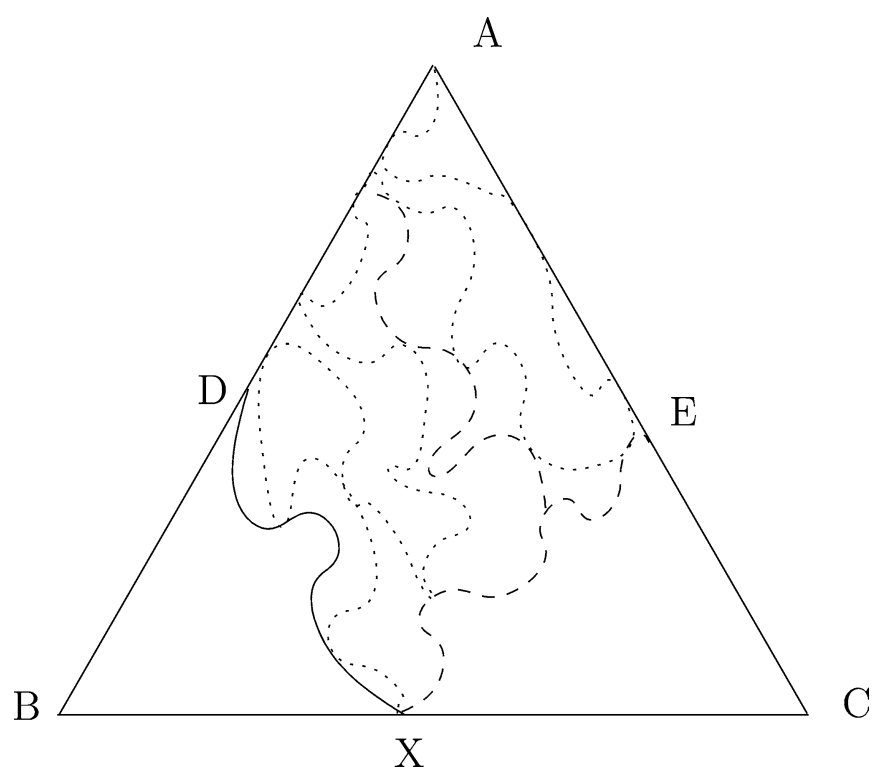

Fig. 5. The - stylized - exploration process (dotted).

(dashed), and the upper-half of $A C$ is either crossed by a white path from $A D$ to $X E$ or by a black path from $D X$ to $E A$. The exploration path goes from $A$ to $X$ leaving white vertices on its left and black vertices on its right. In the case where there is a white crossing between $A D$ and $X E$, it is clear that the last edge of $A B C$ visited by the exploration process before it reaches $X$ is $A B$. Conversely, if there is a black crossing between $D X$ and $E A$, the exploration process last visits $A C$.

Smirnov has proved that the exploration process converges to a chordal $\operatorname{SLE}_{6}$ (see [16]). So let $\gamma$ denote the trace of a chordal SLE 6 from $A$ to $B$ in $A B C, \tau$ be the first time it hits $B C$, and $g$ the last time it hits $(A B) \cup(A C)$ before $\tau$. We may now state the SLE interpretation of Watts' formula in the topological rectangle $A B X C$ (the left-hand should be understood as the scaling limit of the corresponding discrete probabilities):

$$
\mathbb{P}(C(A B, X C, b) \cap C(B X, C A, b))=\mathbb{P}\left(\gamma_{\tau} \in B X\right)-2 \int_{B}^{X} \mathbb{P}\left(\gamma_{g} \in A C \mid \gamma_{\tau}=Y\right) \mathbb{P}\left(\gamma_{\tau} \in d Y\right) .
$$

Recall that $\mathbb{P}\left(\gamma_{\tau} \in B X\right)=B X / B C$ (Cardy's formula). Watts' conjectural formula is equivalent to:

$$
\mathbb{P}\left(\gamma_{g} \in A C \mid \gamma_{\tau}=X\right)=\frac{1}{B(2 / 3,2 / 3)} \int_{0}^{F^{-1}(X)} \frac{d t}{(t(1-t))^{1 / 3}},
$$

where $F$ is the conformal equivalence between $(\mathbb{H}, \infty, 0,1)$ and $(A B C, A, B, C)$. Let us explicit the relation between chordal $\mathrm{SLE}_{6}$ in $A B C$ and the $R B M$ we studied in the previous section. As mentioned in the introduction, the hull of chordal $\mathrm{SLE}_{6}$ in $A B C$ from $A$ to $B$ stopped at time $\tau$ and the hull of the RBM in $A B C$ stopped at its first hitting of $B C$ are identical in law. Though, the processes producing these hulls are starkly different (see [10]). Indeed, the SLE trace is non self-traversing, while the RBM is likely to go back through its past hull. Let $Z$ be such a RBM, $\tau$ the first time it hits $B C, g$ the last time before $\tau$ it visits $(A B) \cap(A C)$. We have seen that:

$$
\mathbb{P}\left(Z_{g} \in A C \mid Z_{\tau}=X\right)=\frac{1}{B(2 / 3,2 / 3)} \int_{0}^{F^{-1}(X)} \frac{d t}{(t(1-t))^{1 / 3}}
$$


and this is exactly the formula proposed by Watts for the scaling limit of critical percolation clusters, i.e. SLE6. Note also that the lowest points of the hulls on $A B$, say, have the same law since they are hull measurable. Since $\gamma$ is non self-traversing, $\gamma_{g}$ is either the lowest point on $A B$ or the lowest point on $A C$; but a.s. $Z_{g}$ is not the lowest point on $A B$ or $A C$.

Given these differences between the underlying curve laws, replacing the $\mathrm{SLE}_{6}$ by the reflected Brownian motion in the "last visited edge" probability problem, the answer should a priori be different. Hence, there are two possibilities:

- For some reason, the $\mathrm{SLE}_{6}$ and RBM "last visited edge" probabilities coincide, and Watts' prediction holds.

- These probabilities differ, and Watts' prediction does not hold. ${ }^{1}$

\section{Vases}

In this section, we generalize the previous properties of RBM, replacing wedges by more general domains with a symmetry axis. More precisely, let $h: \mathbb{R}_{+} \rightarrow \mathbb{R}_{+}$be a positive, differentiable function. Suppose $h(0)=0$, and $h(x)>0$ for $x>0$. Then we will consider the "vase" $C_{h}$ :

$$
C_{h}=\{z \in \mathbb{C}: \Re z \geqslant 0,|\Im z| \leqslant h(\Re(z))\} .
$$

The shape function $h$ is fixed in this section, so we may omit the subscript. As in the previous section, we define a tailor-made discrete model to prove an intertwining relation for its continuous limit. The situation here is slightly more complicated, so we will use a continuous time, discrete state space Markov chain. For the sake of simplicity, suppose the function $h$ is strictly increasing. Let $N$ be some large, fixed integer, and let $x_{k}=h^{-1}(k / N)$. We will consider a graph $\Gamma$ (depending on $h, N)$ with vertices $\left(x_{k}+i y / N\right)_{k \in \mathbb{N}, y \in \mathbb{Z},|y| \leqslant k}$. For $k \in \mathbb{N}$, define $\alpha_{k}=\arctan \left(1 /\left(x_{k+1}-x_{k}\right)\right)$. Let us now define the jump rates (non diagonal elements of the $Q$-matrix). If $z=x_{k}+i y / N$ is an inner point of $\Gamma$ (i.e. $|y|<k$ ), the jump rates are:

$$
\left\{\begin{array}{l}
Q(z, z+i)=Q(z, z-i)=\frac{1}{2}, \\
Q\left(z, x_{k+1}+i y / N\right)=\left[\operatorname{cotan} \alpha_{k}\left(\operatorname{cotan} \alpha_{k}+\operatorname{cotan} \alpha_{k-1}\right)\right]^{-1}, \\
Q\left(z, x_{k-1}+i y / N\right)=\left[\operatorname{cotan} \alpha_{k-1}\left(\operatorname{cotan} \alpha_{k}+\operatorname{cotan} \alpha_{k-1}\right)\right]^{-1} .
\end{array}\right.
$$

These jump rates are chosen in order to get a zero mean, isotropic walk. For a boundary point $z=x_{k}+i k / N$ :

$$
\left\{\begin{array}{l}
Q(z, z-i / N)=1 / 2, \\
Q\left(z, x_{k+1}+i k / N\right)=Q\left(z, x_{k+1}+i(k+1) / N\right)=\left[\operatorname{cotan} \alpha_{k}\left(\operatorname{cotan} \alpha_{k}+\operatorname{cotan} \alpha_{k-1}\right)\right]^{-1} .
\end{array}\right.
$$

For the apex, set $Q\left(0, x_{1}\right)=Q\left(0, x_{1} \pm 1 / N\right)>0$. Here the jump rates are chosen so that intertwining relation holds. More precisely, let $\Lambda$ be the Markov transition kernel from $\left(x_{k}\right)_{k \in \mathbb{N}}$ to $\Gamma$ defined as:

$$
\Lambda\left(x_{k}, .\right)=\frac{1}{2 k+1} \sum_{y=-k}^{k} \delta_{x_{k}+i y / N} .
$$

Then the intertwining relation is valid for $Q$-matrices. More precisely, let $Q$ be the $Q$-matrix of the Markov process just defined, and $\tilde{Q}$ be the $Q$-matrix of the Markov process on $\left\{x_{k}\right\}_{k \in \mathbb{N}} \subset \mathbb{R}_{+}$defined by the jump rates:

$$
\left\{\begin{array}{l}
\tilde{Q}\left(x_{k}, x_{k+1}\right)=\frac{2 k+3}{2 k+1}\left[\operatorname{cotan} \alpha_{k}\left(\operatorname{cotan} \alpha_{k}+\operatorname{cotan} \alpha_{k-1}\right)\right]^{-1}, \\
\tilde{Q}\left(x_{k}, x_{k-1}\right)=\frac{2 k-1}{2 k+1}\left[\operatorname{cotan} \alpha_{k-1}\left(\operatorname{cotan} \alpha_{k}+\operatorname{cotan} \alpha_{k-1}\right)\right]^{-1},
\end{array}\right.
$$

\footnotetext{
${ }^{1}$ In fact one can prove rigorously that Watts' prediction is correct, using properties of $S L E_{6}$ (J. Dubédat, Excursion Decompositions for SLE, in preparation).
} 
for $k>0$, and $\tilde{Q}\left(0, x_{1}\right)=3 Q\left(0, x_{1}\right)$. It is immediate to check that:

$$
\Lambda Q=\tilde{Q} \Lambda \text {. }
$$

Then it is sufficient to exponentiate this intertwining relation (in appropriate Banach spaces, say $\operatorname{End}\left(l_{1}(\Gamma)\right)$ and $\left.\operatorname{End}\left(l_{1}\left(\left\{x_{k}\right\}_{k \in N}\right)\right)\right)$ to get the intertwining relation for the associated semigroups $\left(P_{t}\right),\left(\tilde{P}_{t}\right)$ :

$$
\Lambda P_{t}=\tilde{P}_{t} \Lambda
$$

for all positive time $t$. We now examine the scaling limit of the second process. Let $f$ be some arbitrary function in $C_{b}^{2}\left(\mathbb{R}_{+}\right)$. Then, as $N$ goes to infinity, if $k=k_{N}$ is such that $x_{k_{N}} \rightarrow x$ for some $x \in \mathbb{R}_{+}$:

$$
\begin{aligned}
Q f\left(x_{k}\right) & =\frac{1}{(2 k+1)\left(\operatorname{cotan} \alpha_{k}+\operatorname{cotan} \alpha_{k-1}\right)}\left(\frac{2 k+3}{\operatorname{cotan} \alpha_{k}}\left(f\left(x_{k+1}\right)-f\left(x_{k}\right)\right)+\frac{2 k-1}{\operatorname{cotan} \alpha_{k-1}}\left(f\left(x_{k-1}\right)-f\left(x_{k}\right)\right)\right) \\
& =\frac{1}{N^{2}}\left(\frac{h^{\prime}(x)}{h(x)} f^{\prime}(x)+\frac{1}{2} f^{\prime \prime}(x)\right)+\mathrm{o}\left(\frac{1}{N^{2}}\right) .
\end{aligned}
$$

After a time rescaling, these discrete generators "converge" to the diffusion generator:

$$
\frac{h^{\prime}}{h} \frac{\partial}{\partial x}+\frac{1}{2} \frac{\partial^{2}}{\partial x^{2}}
$$

One checks immediately that a scale function for this diffusion on $\mathbb{R}_{+}$is:

$$
\phi(x)=\int_{1}^{x} \frac{1}{h^{2}(u)} d u .
$$

For a wedge, $h(x)=x \tan \alpha$, then $x \mapsto x^{-1}$ is a scale function for the associated diffusion, i.e. the 3-dimensional Bessel process.

We now discuss the proof for the convergence of the processes defined above. It is similar to the previous proof, with added technicalities. Firstly, one has to adapt the submartingale-problem characterization for vases. One possibility is to map conformally $C_{h}$ onto a wedge, say $C_{\pi / 4}$ (for the Riemann Mapping Theorem, see [2]); after a time change (see [13] for a discussion of the image of a complex Brownian motion under a conformal map), one may use a variant of the Varadhan-Williams result. Here the reflection angle may vary along the boundary, but stay negative. Then one argues as above: subsequential scaling limits exist (tightness); they satisfy a submartingale-problem - a martingale problem for the one-dimensional diffusion - , as seen by dominated convergence after applying Dynkin's formula; hence the limit is uniquely determined, and the processes converge weakly. Reflections are taken care of as before, via an occupation time estimate. The intertwining is obviously preserved in the continuous limit. Hence one may state:

Proposition 3. The reflected Brownian motion in the vase $C_{h}$ with constant reflection index -1 is intertwined with its real part, which is a diffusion on $\mathbb{R}_{+}$corresponding to the generator $\frac{h^{\prime}}{h} \frac{\partial}{\partial x}+\frac{1}{2} \frac{\partial^{2}}{\partial x^{2}}$, by the Markov transition kernel from $\mathbb{R}_{+}$to $C_{h}$ :

$$
\Lambda(x, .)=\operatorname{Unif}([x-i h(x) ; x+i h(x)]) .
$$

Let us stress that the reflection angle varies along the boundary; the reflection at $x+i h(x)$ points in the $-i \exp \left(2 i \arctan h^{\prime}(x)\right)$ direction. Starting from an arbitrary diffusion on $\mathbb{R}_{+}$, one may consider an increasing scale function $\phi$. Setting $h(x)=1 / \sqrt{\phi^{\prime}(x)}$, one constructs an intertwined reflected Brownian motion. Of course, one can also consider shape functions defined and positive on $\mathbb{R}$ ("funnels" rather than vases), or on a segment $[a, b]$, with $h(x)=0$ if and only if $x \in\{a, b\}$ (closed vases). We will now consider an example of the previous construction.

Corollary 1. Consider the shape function $h(x)=x^{\beta}$, with $\beta>0$. Then the real part of the RBM in the vase $C_{h}$ with constant reflexion index -1 is a Bessel process of dimension $2 \beta+1$. 
When the shape function $h$ is not monotone, one has to be a bit more cautious about the definition of the grids, but this requires no substantial changes in the proof (the terms $2 k+1$ are to be replaced by $l\left(x_{k}\right)$, with $\left.l\left(x_{k+1}\right)-l\left(x_{k}\right)=2 \operatorname{sgn} h^{\prime}\left(x_{k}\right)\right)$.

\section{Acknowledgements}

I would very much like to thank Wendelin Werner for his help and advice, especially regarding Watts' formula. I would also like to thank Jim Pitman for stimulating discussions on intertwining relations.

\section{References}

[1] M. Abramowitz, I. Stegun (Eds.), Handbook of Mathematical Functions, 1965.

[2] L. Ahlfors, Complex Analysis, third ed., McGraw-Hill, 1979.

[3] H. Bateman, Higher Transcendental Functions, McGraw-Hill, 1953.

[4] P. Biane, Quelques propriétés du mouvement brownien dans un cône, Stochastic Process. Appl. 53 (2) (1994) 233-240.

[5] P. Biane, Intertwining of Markov semi-groups, some examples, in: Séminaire de Probabilités, XXIX, in: Lecture Notes in Math., vol. 1613, Springer, Berlin, 1995, pp. 30-36.

[6] J.L. Cardy, Critical percolation in finite geometries, J. Phys. A: Math. Gen. 25 (1992) 201-206.

[7] P. Carmona, F. Petit, M. Yor, Beta-gamma random variables and intertwining relations between certain Markov processes, Rev. Mat. Iberoamericana 14 (2) (1998) 311-367.

[8] H. Kesten, Percolation Theory for Mathematicians, Birkhaüser, 1982.

[9] G. Lawler, O. Schramm, W. Werner, Values of Brownian intersection exponents. I. Half-plane exponents, Acta Math. 187 (2) (2001) 237-273.

[10] G. Lawler, O. Schramm, W. Werner, Conformal restriction: the chordal case, J. Amer. Math. Soc. 16 (2003) $917-955$.

[11] J.F. Le Gall, Mouvement brownien, cônes et processus stables, Probab. Theory Related Fields 76 (1987) 587-627.

[12] J.F. Le Gall, Une approche élémentaire des théorèmes de décomposition de Williams, in: Séminaire de Probabilités, XX, 1984/1985, in: Lecture Notes in Math., vol. 1204, Springer, Berlin, 1986, pp. 447-464.

[13] D. Revuz, M. Yor, Continuous Martingales and Brownian Motion, Springer, Berlin, 1991.

[14] L.C.G. Rogers, J. Pitman, Markov functions, Ann. Probab. 9 (1981) 573-582.

[15] L.C.G. Rogers, D. Williams, Diffusions, Markov Processes, and Martingales. Volume One: Foundations, second ed., Wiley, 1993.

[16] S. Smirnov, Critical percolation in the plane. I. Conformal Invariance and Cardy's formula II. Continuum scaling limit, 2001, in preparation.

[17] S.R.S. Varadhan, R.J. Williams, Brownian motion in a wedge with oblique reflection, Comm. Pure Appl. Math. 38 (1985) $405-443$.

[18] B. Virág, Brownian beads, Probab. Theory Related Fields 127 (3) (2003) 367-387.

[19] G.M.T. Watts, A crossing probability for critical percolation in two dimensions, J. Phys. A: Math. Gen. 29 (1996) 363-368.

[20] W. Werner, Critical exponents, conformal invariance and planar Brownian motion, in: Proceedings of the 3rd Europ. Congress Math., in: Prog. Math., vol. 202, Birkhäuser, 2001, pp. 87-103. 